\title{
Comparison of Mouth Opening Length and Masseter Thickness in Subjects with and without Temporomandibular Joint Pain
}

\author{
Ye-Jin An, BSc, $\mathrm{PT}^{1}$; Sung-dae Choung, Ph.D, PT ${ }^{1}$; No-Yul Yang, Ph.D, $\mathrm{OT}^{2}$ \\ ${ }^{1}$ Department of Physical Therapy, Division of Health Science, Baekseok University \\ ${ }^{2}$ Department of Occupational Therapy, Chungnam State University
}

Background Although the temporomandibular joint (TMJ) is a bilateral joint, it is often used asymmetrically during active daily living. When there is a biomechanical or physiological imbalance in the masseter muscle, which is used to chew and close the mouth, a TMJ disorder can be occurred.

Purpose The purpose of our study was to compare the extent to which subjects with and without TMJ pain could open their mouths and the asymmetry of masseter thickness.

Study design A cross-sectional study

Methods This study included 15 subjects with TMJ pain and 15 subjects without TMJ pain (control group). The maximum mouth opening and rotation mouth movement were measured using Image $\mathbf{J}$, and masseter thickness was measured using ultrasonography.

Results The results showed that there were significant differences in the maximum mouth opening and the rotation mouth movement between the groups $(p<0.05)$. However, no significant difference was found in the asymmetry of the masseter thickness $(p>0.05)$.

Conclusions The pain felt in the TMJ was not related to the asymmetry of the masseter thickness; however, the maximum mouth opening and reduction of the rotation mouth movement were closely related to the pain level. Therefore, we recommend increasing the rotation mouth movement of the jaw to improve TMJ pain and solve the asymmetry of the rotation mouth movement of the jaw.

Key words Asymmetry; Masseter; Mouth opening; Temporomandibular joint.
J Musculoskelet Sci Technol 2021: 5(2): 54-58 Published Online Dec 31, 2021 pISSN 2635-8573 eISSN 2635-8581
Article History Received 24 Aug 2021 Revised 12 Sep 2021 (1st)
Revised 26 Oct 2021
(2nd)
Accepted 28 Oct 2021
CONTACT
dae282282@bu.ac.kr
Sung-dae Choung,
Department of Physical
Therapy, Division of
Health Science, Baekseok
University

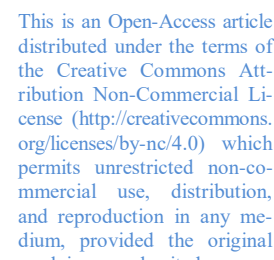
Open-Access article under the terms of he Creative Commons Att- cense (http://creativecommon org/licenses/by-nc/4.0) which ermits unrestricted non-co- and reproduction in any mework is properly cited.

\section{INTRODUCTION}

The temporomandibular joint (TMJ) is a complex lubrication joint between the mandibular fossa of the temporal bone and the articular process of the mandible. The TMJ consists of a synovial membrane, a joint pocket, a joint disc, and bone tissue. Unlike other synovial joints, the articular cartilage of the TMJ is characterized by a layer of connective tissue. ${ }^{1}$ The TMJ is one of the most used joints in the human body, and it moves more than 2,000 times a day. About $40-60 \%$ of the population has a TMJ disorder and has been reported to experience pain and limitation of the
TMJ range of motion. ${ }^{2,3}$ Symptoms of TMJ disorders include a limited range of motion of the mandible, masticatory and joint pain, joint sounds (clicking, popping, or bone fricative sounds), fascia pain, and functional limitations. ${ }^{4}$ The most common symptoms of TMJ disorders are reportedly joint and muscle pain. ${ }^{5}$ TMJ disorders are caused by various and complex factors; the main factors are overactivity of the muscles and abnormal functional activities (e.g., incorrect biting) due to factors such as trauma, emotional stress, and malocclusion. $3,6,7$

The two TMJs are bilateral, and because both sides of the mandible roll or slide together, one TMJ cannot be moved 
without affecting the other. However, chewing food is often performed asymmetrically, ${ }^{8}$ and a study found that about $78 \%$ of the subjects preferred using one side during mastication. ${ }^{9}$ The position and movement of the TMJ is controlled by the masticatory muscles around the joint, including the masseter, temporalis, medial pterygoid, and lateral pterygoid. When biomechanical and physiological imbalance of these muscles occurs, TMJ disorders can be occurred. ${ }^{5,10}$

It has been reported that people with TMJ disorders have a high degree of asymmetry in the masticatory muscles. ${ }^{11}$ In the resting posture, the masseter has the highest asymmetry index compared to the digastric, anterior masseter, anterior temporalis, and sternocleidomastoid. The masseter thickness asymmetry in the resting position is a result of preferring to use one side of the mouth during mastication. Therefore, it is necessary to measure the masseter thickness asymmetry in the resting position to determine whether the asymmetry that appears in people with TMJ disorders is due to pain or muscle weakness.

People with TMJ disorders generally have a reduced mouth-opening range, making it difficult to perform the functional movement of putting food into the mouth. ${ }^{12}$ When they open their mouths and the TMJ exceeds the limited range, inflammation and pain occur. As a result, they restrict their mouth opening range to minimize pain.

The opening of the mouth involves a pure rotational movement until the incisors are $2.0-2.5 \mathrm{~cm}$ apart. After the rotation movement reaches its maximum, a translation movement occurs. ${ }^{3}$ In previous studies, tape measures have mainly been used to measure the range of motion of the mouth. However, this method has been shown to result in a measurement error of $0.2-0.3 \mathrm{~cm}$, and the reliability is not high. ${ }^{13}$ Therefore, in this study, a motion capture device was used to measure rotational and translational movements during TMJ opening in subjects with and without TMJ dysfunction. The aim of this study was to compare the asymmetry of the rotation and translation distance and masseter thickness in subjects with and without TMJ pain.

\section{METHODS}

Subjects

The study details were verbally shared with potential subjects before recruitment. All subjects expressed their willingness to participate, and verbal consent was obtained before subjects participated. This study was approved by the Baekseok University Human Studies Committee (BUIRB202102-HR-043). A total of 15 subjects with TMJ pain made up the TMJ group, and 15 subjects without TMJ pain made up the control group. The subjects' ages ranged from 20 to 26 years, and their average age was 23 years (Table 1). All subjects were classified into the TMJ group and the control group according to their answers to the following two questions. ${ }^{14}$ (1) Have you experienced pain in your temple, face, in front of the ears (TMJ), or jaw at least once within the past week? (2) Have you experienced jaw pain when opening your mouth or chewing food at least once within the past week? Those who answered "yes" to both questions were assigned to the TMJ group, and those who answered "no" to both questions were assigned to the control group. Exclusion criteria included neurological problems, receiving regular dental treatment, experience of trauma around the neck or jaw, and participation in the study.

\section{Measurement}

\section{1) Ultrasonography}

The LOGIQ P6 PRO (GE Inc., New Jersey, USA) was used to measure the masseter muscle thickness. The ultrasonic probe was placed at an angle of $60^{\circ}$ to the skin surface $2.5 \mathrm{~cm}$ above the angle of the mandibular of the subject. ${ }^{15}$ Both masseter muscles were measured three times, and average values were calculated.

\section{2) Image $\mathbf{J}$}

To measure the maximum mouth opening and rotation, the subject was placed on a chair with a backrest, and the distance between the camera (on a tripod) and the nose was fixed at $50 \mathrm{~cm}$. Mouth opening was repeated three times to derive images using Image $\mathbf{J}$ software (Image Processing and Analysis in Java version 1.50i, USA, National Institutes of Health).

\section{Procedure}

\section{1) Measurement of masseter thickness}

The subjects were placed in a sitting position. The examiner asked each subject to maintain a sitting posture with their head upright. ${ }^{15}$ Images of the left and right

Table 1. General characteristics of the subjects

\begin{tabular}{ccc}
\hline & $\begin{array}{c}\text { TMJ group } \\
(\mathrm{n}=15)\end{array}$ & $\begin{array}{c}\text { Control group } \\
(\mathrm{n}=15)\end{array}$ \\
\hline Age $(\mathrm{yr})$ & $23.2 \pm 1.3$ & $22.8 \pm 2.2$ \\
Height $(\mathrm{cm})$ & $166.3 \pm 9.4$ & $166.9 \pm 8.7$ \\
Weight $(\mathrm{kg})$ & $60.7 \pm 12$ & $64.9 \pm 14.3$ \\
\hline Body mass index & $21.8 \pm 2.9$ & $23.1 \pm 4$ \\
\hline
\end{tabular}


masseter muscles were collected with the subject's mouth in a resting position, and a total of three images were saved. To rest the TMJ, the tongue was placed on the hard palate just behind the upper teeth, the upper and lower teeth were naturally spread apart, and the lips were closed and facing forward. ${ }^{17}$ The thickness of the masseter muscles was determined by drawing vertical lines on the muscles, excluding the fascia, in each image (Figure 1).

2) Measurement of maximum and rotation mouth opening

The maximum and rotation mouth opening were measured while the subject sat upright in a chair with a backrest and faced forward. To measure the maximum mouth opening, the mouth was opened as wide as possible, and the distance between the upper and lower teeth was measured. To measure the maximum mouth opening, the examiner asked the subject to "open your mouth as wide as you can".

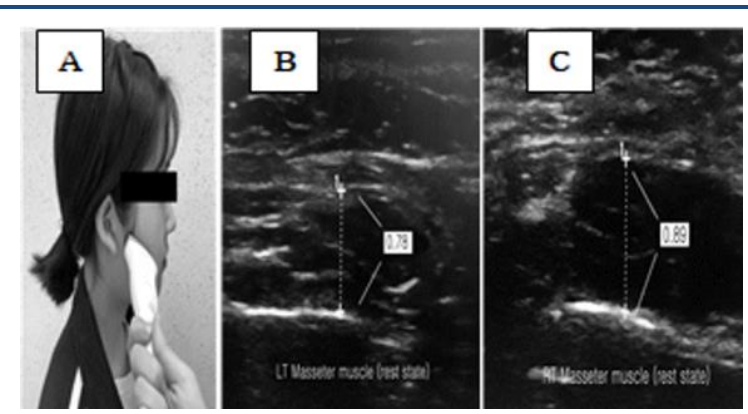

Figure 1. Measurement of masseter muscle thickness using ultrasonography (A: Measuring position. B: Ultrasound image showing left masseter muscle in resting state. C: Ultrasound image showing right masseter muscle in resting state.).
To measure the rotation mouth movement, the examiner asked the subject to "slowly open your mouth until I told them to stop". The examiner palpated the subject's both mandibular condyles with both hands and said "stop" before the condyles were translated forward, the distance between the upper and lower teeth was measured. When the maximum and rotation mouth opening were performed, Image $\mathrm{J}$ was used to take pictures of each subject and perform the measurements (Figure 2).

\section{Data analysis}

SPSS ver. 1.0.0-2843 (SPSS Inc., Chicago, USA) was used for the statistical analysis of the data. The paired $t$-test was used for intra-group analysis, the independent $t$-test was used for inter-group analysis, and the significance level was set at 0.05 .

\section{RESULTS}

Comparison of the maximum and rotation mouth openings

When the maximum and rotation mouth opening of the TMJ group and control group were compared, there was a significant difference between the TMJ group and the control group $(p<0.05)$ (Table 2).

Comparison of the asymmetry index of masseter thickness

The results showed that there was no significant difference in masseter thickness between the TMJ group and the control group (Table 3).

* Asymmetry index $=\frac{\text { masseter thickness in pain side }}{\text { masseter thickness in non }- \text { pain side }}$

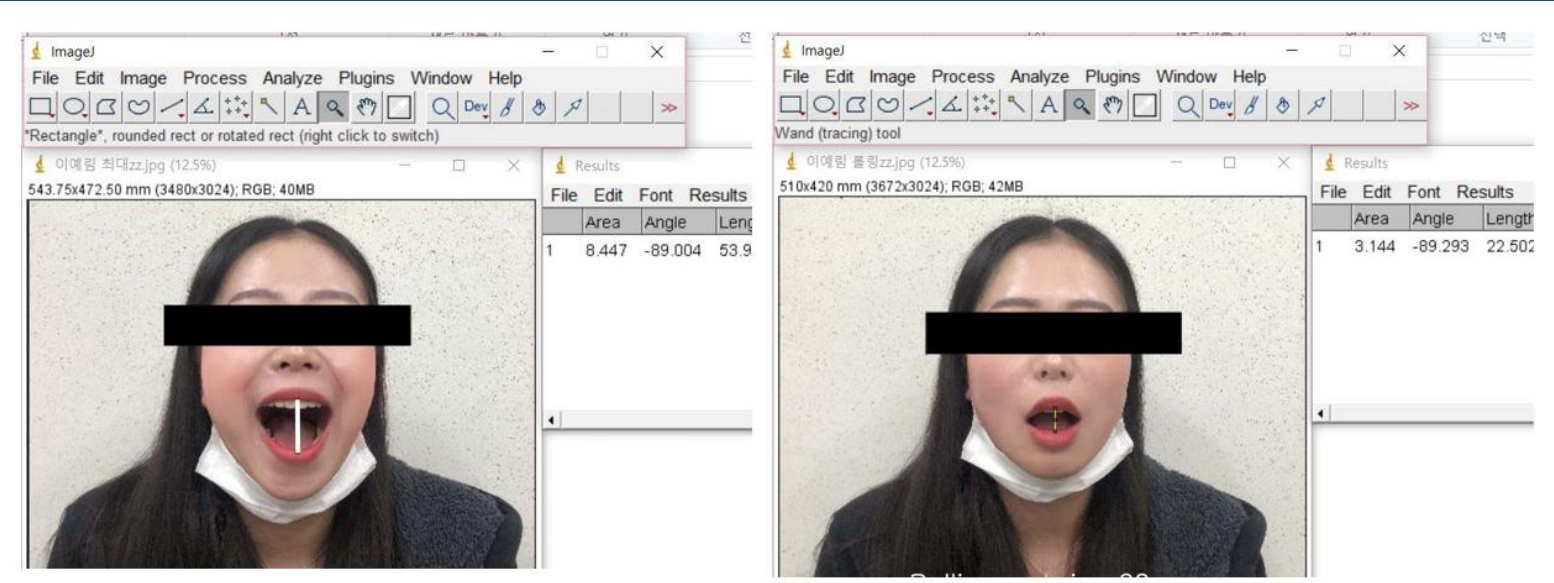

Figure 2. Measurement of mouth opening using Image J (Maximum mouth opening and rotation mouth opening). 
Table 2. Comparison of maximum mouth opening and rotation mouth opening

\begin{tabular}{cccc}
\hline & $\begin{array}{c}\text { TMJ group } \\
(\mathrm{n}=15)\end{array}$ & $\begin{array}{c}\text { Control group } \\
(\mathrm{n}=15)\end{array}$ & $p$ \\
\hline $\begin{array}{c}\text { Maximum mouth } \\
\text { opening }(\mathrm{cm})\end{array}$ & $45.47 \pm 2.36$ & $49.27 \pm 3.02$ & 0.011 \\
$\begin{array}{c}\text { Rotation mouth } \\
\text { opening }(\mathrm{cm})\end{array}$ & $23.80 \pm 2.08$ & $34.13 \pm 3.06$ & 0.012 \\
\hline
\end{tabular}

Table 3. Comparison of the asymmetry index of masseter thickness

\begin{tabular}{cccc}
\hline & $\begin{array}{c}\text { TMJ group } \\
(\mathrm{n}=15)\end{array}$ & $\begin{array}{c}\text { Control group } \\
(\mathrm{n}=15)\end{array}$ & $p$ \\
\hline $\begin{array}{c}\text { Masseter } \\
\text { asymmetry index* }\end{array}$ & 0.98 & 1.06 & 0.447 \\
\hline
\end{tabular}

\section{DISCUSSION}

Temporomandibular disorders (TMD) are the most common pain syndromes in the oral and maxillofacial regions and may be associated with the bilateral TMJ, the mastication muscles, and the blood vessels and nerves distributed in these tissues. The major symptoms of TMD include masticatory muscle and TMJ pain, TMJ sounds, and restriction of mandibular movement, and related symptoms include ear pain, stiffness, tinnitus, dizziness, neck pain, and headache. ${ }^{18,19,20}$ The frequency of pain in the TMJ tends to increase with modern people's exposure to poor eating habits, lifestyle changes, and social and psychological problems.

The purpose of this study was to investigate the length of maximum and rotation mouth opening and masseter thickness according to the presence or absence of TMJ pain. It was found that there was a significant difference in mouth opening between the TMJ group and the control group in this study. The rotation movement of the TMJ takes place until the incisors are $2.0-2.5 \mathrm{~cm}$ apart, and then a sliding or translation movement is performed. ${ }^{3}$ Giro et al. (2016) suggested that damage to the mandibular muscle and joint impairment may limit the range of motion. ${ }^{21}$ In addition, changes in the TMJ and neuromuscular control affect pain, which alters the direction and precision of TMJ movement.

In this study, the TMJ group subjects opened their mouths an average of $3.4 \mathrm{~cm}$, demonstrating a limited opening compared to the normal opening of $5 \mathrm{~cm}$. However, this value was within the functional opening range $(3.75$ $\mathrm{cm}$ ). Therefore, the subjects in the TMJ group used their TMJs functionally, and they could be considered as expe- riencing mild TMJ disorders rather than severe difficulties in their daily lives.

There was no significant difference in the asymmetry of the masseter thickness. We thought that a patient with mild TMJ pain did not show any asymmetry of the masseter thickness, and pain or weakening of the masseter. In previous studies, patients with TMJ pain had an increased thickness of the masseter muscle. Ariji et al. (2004) reported that patients with TMJ pain exhibited a thickening of the masseter muscle that was associated with muscle edema. ${ }^{22}$ However, similar to this study, others have reported that there is no statistically significant difference in the thickness of the muscles according to the presence or absence of TMJ pain. ${ }^{23}$ Therefore, although masseter asymmetry is not closely related to TMJ pain, it is thought that the decrease in rotation mouth opening is closely related.

The limitations of this study are as follows. This study recruited subjects with and without TMJ pain. With the average mouth opening range of the TMJ group being 3.4 $\mathrm{cm}$, this group does not represent patients with TMJ disorders with severe pain. Therefore, it is difficult to generalize the results of this study to all patients with TMJ pain. Also, since this study was a cross-sectional experiment, we could not observe subjects in the long term. Therefore, in future studies, more subjects and patients with a range of TMJ pain levels should be studied prospectively.

\section{CONCLUSION}

The purpose of this study was to compare the length of mouth opening and asymmetry of masseter thickness between the TMJ and healthy group. It was found that there was a significant difference in the amount of rotation and translation opening between the groups. Therefore, exercise programs for patients with TMJ pain should include efforts to increase the amount of rotation and translation opening without TMJ pain. In contrast, there was no significant difference in the asymmetry of the masseter thickness.

\section{Key Points}

Question Is there a difference in the asymmetry of the mouth opening and masseter thickness in people with and without temporomandibular joint pain?

Findings There was a significant difference in the maximum mouth opening and the rotation movement between people with and without temporomandibular joint pain. However, no significant difference was found in the asymmetry of masseter thickness between the groups. 
Meaning These finding suggest that if there is pain in the TMJ, there is a difference in masseter thickness asymmetry and mouth opening.

Article information

Conflict of Interest Disclosures: None.

Funding/Support: None.

Acknowledgment: None.

Ethic Approval: This study was approved by the Baekseok University Human Studies Committee (BUIRB202102-HR-043).

\section{REFERENCES}

1. Kwon YD, Lim YE, Song YS. Relationship between temporomandibular joint (TM) disorder and meridian. $J$ Oriental Rehab Med. 2000;10(10):1-10.

2. Bae Y, Park Y. The effect of relaxation exercises for the masticator muscles on temporomandibular joint dysfunction. J Phys Ther Sci. 2013;25(5):583-586.

3. Okeson JP. Management of temporomandibular disorders and Occlusion. 7th ed. St Louis, MO: Mosby/Elsevier; 2011:32-177.

4. Jung HD, Kim SY, Park HS, et al. Orthognathic surgery and temporomandibular joint symptoms. Maxillofac Plast Reconstr Surg. 2015;37(1):14.

5. Vahedifar M. Temporomandibular joint clinical considerations for practice. Raleigh, NC: Lulu; 2015.

6. Manfredini D, Landi N, Romagnoli M, et al. Etiopathogenesis of parafunctional habits of the stomatognathic system. Minerva Stomatol. 2003;52(8):345-349.

7. Rauhala K, Oikarinen KS, Raustia AM. Role of temporomandibular disorders in facial pain: occlusion, muscle and TMJ pain. Cranio. 1999;17(4):254-261.

8. Ferrario VF, Sforza C, Miani A Jr, et al. Open-close movements in the human temporomandibular joint: does a pure rotation around the intercondylar hinge axis exist? J Oral Rehabil. 1996;23(6):401-408.

9. Pond LH, Barghi N, Barnwell GM. Occlusion and chewing side preference. J Prosthet Dent. 1986;55(4):498-500.

10. Romero-Reyes M, Uyanik JM. Orofacial pain management: current perspectives. J Pain Res. 2014;7:99-115.

11. Emshoff R, Bertram S, Strobl H. Ultrasonographic cross-sectional characteristics of muscles of the head and neck. Oral Surg Oral Med Oral Pathol Oral Radiol
Endod. 1999;87(1):93-106.

12. Muller L, van Waes H, Langerweger $C$, et al. Maximal mouth opening capacity: percentiles for healthy children 4-17 years of age. Pediatr Rheumatol Online J. 2013; 11:17.

13. Walker N, Bohanannon RW, Cameron D. Discriminant validity of temporomandibular joint range of motion measurement obtained with a ruler. J Orthop Sports Phys Ther. 2000;30(8):484-492.

14. Nilsson IM, List T, Drangsholt M. The reliability and validity of self-reported temporomandibular disorder pain in adolescents. J Orofac Pain. 2006;20(2):138-144.

15. Aldemir K, Ustuner E, Erdem E, et al. Ultrasound evaluation of masseter muscle changes in stabilization splint treatment of myofascial type painful temporomandibular diseases. Oral Surg Oral Med Oral Pathol Oral Radiol. 2013;116(3):377-383.

16. Fricton J. Myogenous temporomandibular disorders: diagnostic and management considerations. Dent Clin North Am. 2007;51(1):61-83.

17. Kisner C, Colby LA. Therapeutic exercise: foundations and techniques. 6th ed. Philadelphia, PA: F.A. Davis Co.; 2012.

18. Speciali JG, Dach F. Temporomandibular dysfunction and headache disorder. Headache. 2015;55(Suppl 1): 72-83.

19. Kim ST. TMD recipe made ridiculously simple. Seoul: Publisher Well; 2015.

20. Kaplan AS, Assael LA. Temporomandibular disorders: diagnosis and treatment. W.B.Saunders Co, Inc., 1991.

21. Giro G, Policastro VB, Scavassin PM, et al. Mandibular kinesiographic pattern of women with chronic TMD after management with educational and self-care therapies: a double-blind, randomized clinical trial. J Prosthet Dent. 2016;116(5):749-755.

22. Ariji Y, Sakuma S, Izumi M, et al. Ultrasonographic features of the masseter muscle in female patients with temporomandibular disorder associated with myofascial pain. Oral Surg Oral Med Oral Pathol Oral Radiol Endod. 2004;98(3):337-41.

23. Pereira LJ, Gaviao MB, Bonjardim LR, et al. Muscle thickness, bite force, and craniofacial dimensions in adolescents with signs and symptoms of temporomandibular dysfunction. Eur J Orthod. 2007;29(1):7278. 